03,08

\title{
Возможности моделирования из первых принципов электрон-фононной релаксации и транспортных свойств на примерах оксида кадмия и титаната стронция
}

\author{
(C) В.П. Жуков ${ }^{1}$, Е.В. Чулков ${ }^{2,3}$ \\ ${ }^{1}$ Институт химии твердого тела УрО РАН, \\ Екатеринбург, Россия \\ ${ }^{2}$ Санкт-Петербургский государственный университет, \\ Санкт-Петербург, Россия \\ ${ }^{3}$ Dpto. de Polímeros y Materiales Avanzados: Física, Química y Tecnología, Facultad de Ciencias Químicas, \\ Aptdo. 1072, 20018, San Sebastián, España \\ E-mail: Zhukov@ihim.uran.ru
}

Поступила в Редакцию 2 декабря 2021 г.

В окончательной редакции 20 декабря 2021 г.

Принята к публикации 20 декабря 2021 г.

\begin{abstract}
Первопринципными методами, основанными на теории функционала электронной плотности и его возмущений, теории Больцмана и многочастичной теории электрон-фононного взаимодействия выполнены расчеты времени электронной релаксации, коэффициента Зеебека и проводимости для оксида кадмия с кислородными вакансиями и титаната стронция, допированного ниобием. Показано, что расчеты времени релаксации на основе многочастичной теории приводят к существенно более точным результатам по транспортным характеристикам, чем в случае стандартного приближения постоянного времени релаксации. Показано, что существенное влияние на проводимость оказывает взаимодействие с дефектами.
\end{abstract}

Ключевые слова: оксид кадмия, титанат стронция, электронная структура, метод PAW, теория Больцмана, транспортные характеристики.

DOI: 10.21883/FTT.2022.04.52181.249

\section{1. Введение}

Последнее десятилетие ознаменовалось существенными успехами в развитии методов моделирования термоэлектрических свойств твердых тел „из первых принципов“. На основе теорий Больцмана, теории функционала электронной плотности и многочастичной теории конденсированного состояния был развит математический аппарат для расчета электрон-фононного (ЭФ) взаимодействия [1,2], проводимости электронов, мобильности, коэффициентов Холла и Зеебека, теплопроводности [3-5], характеристик сверхпроводимости и динамики электрон-фононной релаксации [6]. С использованием развитых подходов были реализованы программы, позволяющие выполнять расчеты указанных характеристик твердых тел на базе ранее развитых программных комплексов расчета электронной зонной структуры [7-11]. В случае металлов и недопированных полупроводников были выполнены многочисленные расчеты указанных характеристик, показавшие разумное согласие с экспериментальными данными (см. обзоры [12-17]).

Однако остаются неясными перспективы использования развитых подходов в расчетах свойств допированных полупроводников или сплавов. Известно лишь немного работ, в которых были проведены расчеты для полупроводников с разными концентрациями допирующих элементов $[18,19]$. При этом использовался подход, основанный на теории Больцмана с фиксированным временем ЭФ-релаксации $[3,4]$ без сопоставления с экспериментальными данными. Приближение фиксированного времени ЭФ-релаксации существенно сокращает необходимые для расчетов компьютерные ресурсы, поэтому его использование является стандартным приемом. Однако исследования, проведенные для допированных полупроводников, не позволяют судить о точности получаемых результатов, поэтому нужны дополнительные усилия в данном направлении.

$\mathrm{B}$ силу широкого использования как $\mathrm{CdO}$, так и $\mathrm{SrTiO}_{3}$ во многих направлениях современной техники, данные соединения привлекают значительное внимание исследователей, поэтому был выполнен большой ряд работ по изучению их свойств. В исходном состоянии данные объекты являются полупроводниками с шириной запрещенной щели $0.8 \mathrm{eV}$ для $\mathrm{CdO}$ и $3.25 \mathrm{eV}$ для $\mathrm{SrTiO}_{3}$, однако путем введения вакансий в кислородную подрешетку или гетеровалентного допирования удается перевести их в состояние проводников $n$-типа. Экспериментальные исследования транспортных характеристик $\mathrm{CdO}$ были проведены в работах [20-22], а аналогичные исследования для $\mathrm{SrTiO}_{3}$ - в работах [23-25]. В [20-22] проводимость в $\mathrm{CdO}$ достигалась за счет образования кислородных вакансий при высокотемпературном отжиге, тогда как для $\mathrm{SrTiO}_{3}$ в работах [23-25] - за счет 
допирования $\mathrm{Ta}, \operatorname{Pr}$ или $\mathrm{Nb}$. Были получены зависимости коэффициента Зеебека, проводимости, теплопроводности, концентрации носителей и мобильности носителей от температуры отжига и степени допирования. Данные работы содержат сведения, достаточные для проверки корректности первопринципных методов расчета транспортных свойств, что является главной целью данной работы.

В настоящей работе мы приводим результаты расчетов коэффициента Зеебека и проводимости, выполненные в рамках двух подходов, реализованных в программном комплексе BoltzTraP-1 [3], и в новейшем комплексе PERTURBO [6]. Данные расчеты позволяют оценить точность вычислений транспортных характеристик, выявить достоинства и недостатки подходов и наличие проблем, подлежащих решению.

\section{2. Основы теории}

Первопринципные методы расчета транспортных характеристик твердых тел основаны на сочетании больцмановской феноменологической теории транспорта и вытекающих из многочастичной теории твердых тел методов расчета рассеяния носителей тока. Современное состояние теории Больцмана подробно описано в литературе [5,26-28]. Основы методов расчета рассеяния носителей тока на фононах освещены в работах $[1,2]$. Компьютерные коды для расчета транспортных свойств используют ранее развитые методы расчета электронной зонной структуры на базе теории функционала электронной плотности (ФЭП) [29] и фононов на базе теории возмущений ФЭП [1]. Принципы построения кодов описаны в работах $[3,4,6,10]$, поэтому мы остановимся лишь на некоторых моментах теории, важных для обсуждения полученных нами результатов.

Согласно первому уравнению Онзагера [30] плотность тока в конденсированной среде $j$ связана с создаваемыми внешними источниками напряженностью электрического поля $E$ и градиентом температуры $\nabla T$ соотношением

$$
j=\sigma E-K \nabla T
$$

Предполагается, что в твердом теле напряженность и градиент могут быть не коллинеарны току, т.е. $\sigma$ и $K-$ тензорные величины. Для однородной среды коэффициент Зеебека стандартным образом определяется как коэффициент пропорциональности между напряженностью электрического поля и градиентом температуры, т.е. $E=S \nabla T$, при условии отсутствия тока. Тогда обобщением коэффициента Зеебека, согласно (1), будет тензорная величина

$$
S=\sigma^{-1} K
$$

Связь между $\sigma, K, S$ и электронной структурой материала устанавливается на основе теории Больцмана. Основным предположением в данной теории является то, что функция распределения электрона в материале $f(r, k, t)$ при малой напряженности и малом градиенте температуры лишь незначительно отличается от стационарной функции Ферми.

$$
f_{0}(r, k)=\frac{1}{e^{[\varepsilon(k)-\mu] / k_{\mathrm{B}} T(r)}+1} .
$$

Здесь от координаты зависит только температура, тогда как фиксированный химический потенциал зависит только от свойств материала. Другим предположением теории является так называемое приближение времени релаксации (RTA), в котором считается, что если внешние поля включаются в момент времени $t=0$, то при их снятии в момент $t$ отклонение функции распределения от стационарной изменяется по закону

$$
f(r, k, t)=\left[f(r, k, 0)-f_{0}(r, k)\right] e^{-t / \tau(k)},
$$

т.е. функция распределения возвращается в исходное состояние со скоростью, определяемой характерным временем релаксации $\tau(k)$. В данном приближении решение уравнения Больцмана приобретает вид, см. [28],

$$
f(r, k)=f_{0}(r, k)-\frac{\partial f_{0}}{\partial \varepsilon} \tau(k) v(k)\left[-e E+\frac{\varepsilon(k)-\mu}{T} \nabla T\right],
$$

где $v(k)$ - групповая скорость электрона в состоянии $\varepsilon(k)$.

Ток, переносимый электронами, с учетом того, что стационарная функция распределения не дает в него вклада и без учета вклада от градиента температуры, может быть вычислен как

$$
\begin{aligned}
j & =\frac{e}{4 \pi^{3}} \int d^{3} k v f(r, k) \\
& =-\frac{e^{2}}{4 \pi^{3}} \int d^{3} k \tau(k)\left(-\frac{\partial f_{0}}{\partial \varepsilon}\right)[v \otimes v] E .
\end{aligned}
$$

Т.е. тензор удельной электропроводности записывается как [5]:

$$
\sigma=-\frac{e^{2}}{4 \pi^{3}} \int d^{3} k \tau(k)\left(-\frac{\partial f_{0}}{\partial \varepsilon}\right)[v \otimes v] E .
$$

С учетом же вклада в функцию распределения от градиента температуры полный ток может быть представлен в виде

$$
j=\sigma E+\frac{1}{4 \pi^{3}} \int d^{3} k v\left(-\frac{\partial f_{0}}{\partial \varepsilon}\right) \tau(k) \frac{\varepsilon-\mu}{T}(v \nabla T) .
$$

Отсюда следует, что если ввести тензор,

$$
L^{(n)}=\frac{1}{4 \pi^{3}} \int d^{3} k\left(-\frac{\partial f_{0}}{\partial \varepsilon}\right) \tau(k)(\varepsilon-\mu)^{n}[v \otimes v],
$$

как это обычно делается в теории транспорта, то выражения для тензоров приобретают вид

$$
\begin{gathered}
\sigma=L^{(0)}, \\
S=\left[L^{(0)}\right]^{-1} L^{(1)} \frac{1}{T}, \\
K=L^{(1)} \frac{1}{T} .
\end{gathered}
$$


Если предположить, что время релаксации не зависит от энергии и волнового вектора, то тензор Зеебека становится независимым от времени. Данное предположение положено в основу кодов $[3,4]$, в результате чего их быстродействие радикально увеличивается. Однако ниже мы покажем, что введение этого приближения заметно понижает точность расчетов, по крайней мере для рассматриваемых нами соединений. При этом зависимость $\sigma$ от $\tau$ сохраняется, поэтому в литературе результаты обычно представляются в виде $\sigma / \tau$.

Недавно в кодах $[10,16]$ были реализованы расчеты „из первых принципов“ электрон-фононного вклада (ЭФВ) во время релаксации на базе теории Мигдала-Элиашберга [31,32]. В данной теории считается, что релаксация электрона в состоянии $\left|\psi_{n, \mathbf{k}}\right\rangle$, где $n$-номер ветви зонной структуры, происходит за счет переходов в состояния $\left|\psi_{m, \mathbf{k}+\mathbf{q}}\right\rangle$ вследствие поглощения или эмиссии фононов с волновыми векторами q и энергией $\omega_{\mathbf{q} v}=\varepsilon_{m, \mathbf{k}+\mathbf{q}}-\varepsilon_{n, \mathbf{k}}$. В первом порядке теории возмущений вероятности переходов определяются матричными элементами

$$
g_{m n, v}(\mathbf{k}, \mathbf{q})=\left\langle\psi_{m, \mathbf{k}+\mathbf{q}}\left|\partial_{\mathbf{q} v} V\right| \psi_{n, \mathbf{k}}\right\rangle
$$

где $\partial_{\mathbf{q} \nu} V-$ производная от самосогласованного потенциала по фононному возмущению с волновым вектором q и индексом поляризации $v$. Методы расчета матричных элементов на основании теории возмущений ФЭП в базисе плоских волн описаны в обзоpe [33], а в базисе локализованных функций Ваннье в работах $[1,6,10]$.

Современные реализации теории электрон-фононного взаимодействия основаны на формализме оператора собственной энергии многочастичной теории твердого тела. В этом подходе в рамках приближения RTA $[6,11]$ изменение энергии электронного состояния вследствие электрон-фононного взаимодействия и ширина состояния определяются, соответственно, вещественной и мнимой частями оператора собственной энергии $\Sigma_{n \mathbf{k}}=\Sigma_{n \mathbf{k}}^{\prime}+i \Sigma_{n \mathbf{k}}^{\prime \prime}$, который может быть вычислен как [11]:

$$
\begin{aligned}
\sum_{n \mathbf{k}}= & \sum_{\mathbf{q} v} w_{\mathbf{q}}\left|\mathbf{g}_{m n, v}(\mathbf{k}, \mathbf{q})\right|^{2}\left[\frac{\mathbf{n}\left(\omega_{\mathbf{q} v}\right)+\mathbf{f}_{0}\left(\varepsilon_{m, \mathbf{k}+\mathbf{q}}\right)}{\varepsilon_{n, \mathbf{k}}-\varepsilon_{m, \mathbf{k}+\mathbf{q}}-\omega_{\mathbf{q} v}-\mathbf{i} \eta}\right. \\
& \left.+\frac{\mathbf{n}\left(\omega_{\mathbf{q} v}\right)+1-\mathbf{f}_{0}\left(\varepsilon_{m, \mathbf{k}+\mathbf{q}}\right)}{\varepsilon_{\mathbf{n}, \mathbf{k}}-\varepsilon_{m, \mathbf{k}+\mathbf{q}}+\omega_{\mathbf{q} v}-\mathbf{i} \eta}\right],
\end{aligned}
$$

где $n\left(\omega_{\mathbf{q} v}\right)-$ функция распределения Бозе-Эйнштейна. Тогда, в соответствии с принципом неопределенности „время-энергия“, время релаксации электронного состояния вследствие взаимодействия с фононами может быть вычислено как

$$
\tau_{n \mathbf{k}}(T)=\frac{1}{2 \Sigma_{n \mathbf{k}}^{\prime \prime}(T)} .
$$

Вычисления $\tau_{n \mathbf{k}}$ были реализованы в кодах $[5,6,10]$. Основная проблема таких вычислений, заключается в том, что они требуют гораздо больших компьютерных ресурсов, чем вычисления с фиксированными $\tau$, что связано в первую очередь с необходимостью вычислять фононные состояния с набором волновых векторов распределенных в неприводимой части зоны Бриллюэна. Поэтому расчеты транспортных свойств в этом подходе оказываются возможными для систем, относительно простых в сравнении с системами, доступными в расчетах с фиксированными $\tau$. Однако ниже мы покажем, что вычисления с $\tau$,из первых принципов“, по крайней мере для рассматриваемых нами соединений, приводят к заметно более точным результатам.

\section{3. Детали расчетов}

Расчеты электронной зонной структуры выполнялись для ГЦК-структуры $\mathrm{CdO}$ и простой кубической структуры $\mathrm{SrTiO}_{3}$ псевдо-потенциальным методом в базисе плоских волн, реализованным в программном комплексе Quantum Espresso [8]. Первоначально расчеты проводились для 16 волновых векторов в неприводимой части зоны Бриллюэна (НЧЗБ) в случае $\mathrm{CdO}$ и для 10 векторов для $\mathrm{SrTiO}_{3}$. В обоих случаях в представлении зонных состояний были использованы плоские волны с энергией до $80 \mathrm{Ry}$, a в представлении электронной плотности - до 600 Ry. Известно, что расчеты фононов, выполненные с использованием теории возмущений ФЭП чувствительны к виду используемых псевдопотенциалов (ПП), в особенности для низкоэнергетических фононов. Поэтому нами были опробованы 7 типов ПП. Расчеты были проведены с псевдопотенциалами типа PAW по Крессе-Жоберу (KJPAW) [34] при обменнокорреляционной части по Пердью-Бурке-Эрцзенхфу (PBE) [35] и аналогичному ПП для твердого тела (PBESOL) [36]. Также использовались ультрамягкие ПП по рецепту Раппе-Рабе-Качирас-Джоннопулос (RRKJUS) [37] с обменно-корреляционной частью типа PBE, PBESOL [29,30], а также ПП по Вандербильду (VAN) [38] с обменом-корреляцией по Пердью-Вангу

Вычисленные и экспериментальные значения фононных частот для Г-точки зоны Бриллюэна $\mathrm{SrTiO}_{3}$ и $\mathrm{CdO}$

\begin{tabular}{c|c|c}
\hline \multicolumn{3}{c}{$\mathrm{SrTiO}_{3}$} \\
\hline $\begin{array}{c}\text { Данная } \\
\text { работа }\left(\mathrm{cm}^{-1}\right)\end{array}$ & $\begin{array}{c}\text { Вычисленные ранее } \\
{[40,41]\left(\mathrm{cm}^{-1}\right)}\end{array}$ & $\begin{array}{c}\text { Экспериментальные - } \\
\text { см. }[40,41]\left(\mathrm{cm}^{-1}\right)\end{array}$ \\
\hline 66 & 50 & 42,91 \\
\hline 165 & 160,170 & 170,175 \\
\hline 518 & 562 & 545,547 \\
\hline \multicolumn{3}{|c}{$\mathrm{CdO}$} \\
\hline 485 & 267 & 265 \\
\hline
\end{tabular}


(PW) [39]. На основе расчетов электронных состояний методом теории возмущений ФЭП [8] были проведены расчеты фононных состояний. К наилучшим результатам, частично показанным в таблице, для СdO привели расчеты с PAW ПП по методу $\mathrm{PBE}-\mathrm{KJPAW}$, а для $\mathrm{SrTiO}_{3}$ - с ультрамягкими ПП по методу $\mathrm{PW}-\mathrm{PBE}$. Сопоставление с результатами, полученными в предыдущих расчетах аналогичными методами (коды Quantum Espresso [40] и Abinit [41]) показывает точность расчета, типичную для методов теории возмущений ФЭП.

Однако, как и большинство расчетов электронных зонных состояний на базе теории ФЭП, наши расчеты дают заниженные значения ширины запрещенной зоны: для $\mathrm{SrTiO}_{3}$ она оказывется равной $1.84 \mathrm{eV}$, а для $\mathrm{CdO}$ мы наблюдаем перекрытие состояний вблизи потолка валентной зоны с состояниями зоны проводимости. Ниже будет показано, что для $\mathrm{SrTiO}_{3}$, т.е. при наличии в расчетах запрещенной щели, данная погрешность, повидимому, не приводит к осложнениям при расчетах транспортных характеристик. Однако для оксида кадмия перекрывание валентной зоны с зоной проводимости дает неправильные результаты: в противоречие эксперименту, коэффициент Зеебека оказывается положительным при всех рассмотренных концентрациях носителей тока. Для устранения этой погрешности зонные состояния $\mathrm{CdO}$ были пересчитаны путем введения одноцентровой хаббардовской поправки с параметром $U$ методом, описанным в [42], к обменно-корреляционному потенциалу для $2 p$-орбиталей атома кислорода. При значении $U=6 \mathrm{eV}$ ширина запрещенной зоны, $0.91 \mathrm{eV}$, хорошо соответствует экспериментальным данным; одновременно введение этой поправки устраняет проблему в расчетах коэффициента Зеебека. В соответствии с предыдущими экспериментальными и расчетными данными [35,43-45], запрещенная зона оказывается непрямой, между точками $L$ и $\Gamma$ для $\mathrm{CdO}$ и $R-\Gamma$ для $\mathrm{SrTiO}_{3}$. Полученные в базисе плоских волн дисперсионные кривые электронных состояний для $\mathrm{CdO}$ и $\mathrm{SrTiO}_{3}$ показаны на рис. 1 и 2. При всех изученных в экспериментах [22-25] концентрациях носителей тока, от $\sim 1 \cdot 10^{19}$ до $\sim 15 \cdot 10^{19} 1 / \mathrm{cm}^{3}$, уровень Ферми находится вблизи дна зоны проводимости. $\mathrm{B}$ случае $\mathrm{CdO}$ состояния зоны проводимости образованы $5 s$ Cd-орбиталями, а в случае $\mathrm{SrTiO}_{3}-3 d$ Тi-орбиталями. Для $\mathrm{CdO}$ уровень Ферми пересекает одна ветвь зонных состояний, а для $\mathrm{SrTiO}_{3}-$ одна ветвь состояний вблизи точки $X$ и три ветви вблизи точки Г.

На основании расчетов электронных и фононных состояний были выполнены расчеты характеристик транспорта в $\mathrm{CdO}$ и $\mathrm{SrTiO}_{3}$ методами, описанными в работе [3], программа BoltzTaP-1, и в работе [6], программа Perturbo. Проблема расчета транспортных свойств, как это неоднократно указывалось ранее $[3,4,10]$, состоит в том, что они требуют вычисления большого числа зонных состояний вблизи уровня Ферми, для количества волновых векторов не менее 100000 на полную зону Бриллюэна. В методе [3] данная проблема решается

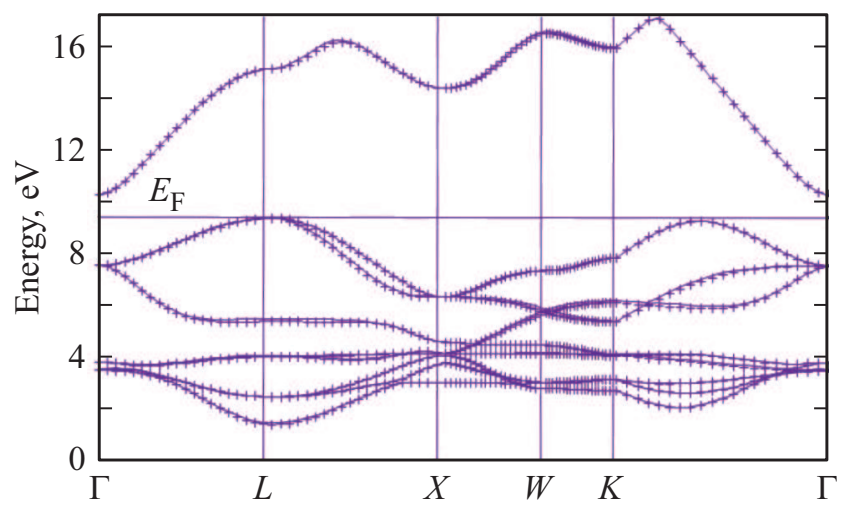

Рис. 1. Дисперсионные кривые для главных направлений в зоне Бриллюэна оксида кадмия. Крестиками отмечены результаты расчетов в базисе плоских волн (программа Quantum Espresso). Сплошные линии соответствуют зонным состояниям, вычисленным в базисе функций Ваннье по методике [46].

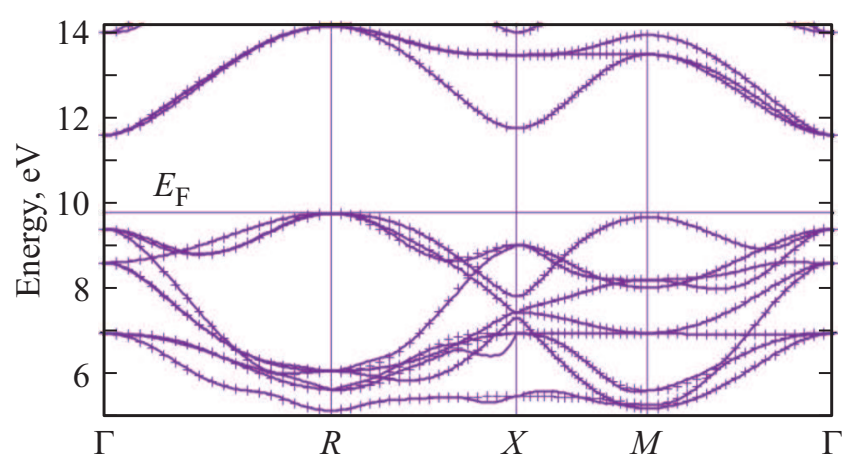

Рис. 2. Дисперсионные кривые для зонных состояний титаната стронция. Сплошные линии соответствуют результатам расчетов в базисе Ваннье [40], а крестиками отмечены зонные состояния, полученные в базисе плоских волн.

путем Фурье-интерполяции между состояниями, полученными в базисе плоских волн.

Аналогичная проблема, в более поздних методах $[6,10,11]$, решается переходом от базиса блоховских функций к базису локализованных функций Ваннье по методам, описанным в [46]. С использованием базиса Ваннье можно получать набор зонных состояний для очень большого числа волновых векторов в зоне Бриллюэна. Очевидно, что состояния, получаемые в базисе Ваннье, должны хорошо соответствовать их аналогам, получаемым в базисе плоских волн. Существенную роль при этом играет выбор стартовых локализованных состояний, которые в итерационных процессах, описанных в [46], преобразуются в базис Ваннье. В наших случаях стартовыми состояниями для $\mathrm{CdO}$ выбирались $5 s-, 5 p$-состояния атомов кадмия и $2 s-, 2 p$-состояния атомов кислорода. Для $\mathrm{SrTiO}_{3}$ в качестве стартового базиса использовались $3 d$ Ті- и $2 p$ О-состояния. Из рис. 1 следует, что энергии состояний $\mathrm{CdO}$ в базисе Ваннье практически не отличаются от энергий в базисе плоских волн. Качество зонных состояний, получаемых 

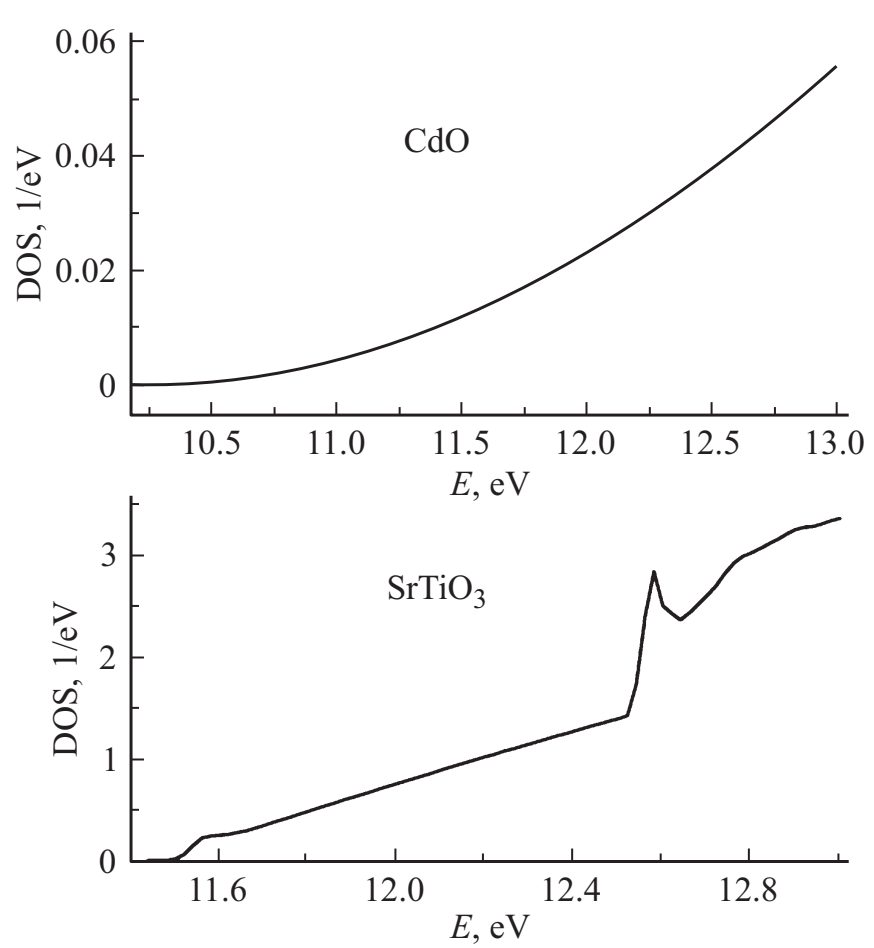

Рис. 3. Плотности состояний вблизи дна зоны проводимости $\mathrm{CdO}$ и $\mathrm{SrTiO}_{3}$.

в базисе Ваннье для $\mathrm{SrTiO}_{3}$, характеризуется рис. 2 . Из него следует, что в верхней части валентной зоны, от 7.5 до $10 \mathrm{eV}$, и в части зоны проводимости от 11.5 до $14 \mathrm{eV}$, энергии состояния, получаемые в базисе Ваннье, практически совпадают с энергиями их аналогов в базисе плоских волн. Некоторые различия имеются для волновых векторов около точки $X$ в диапазоне от 5 до $7 \mathrm{eV}$, однако они несущественны в расчетах транспортных свойств. Таким образом, подтверждается правильный выбор базиса Ваннье. Поэтому в расчетах транспортных свойств как для $\mathrm{CdO}$ так и для $\mathrm{SrTiO}_{3}$ использовались зонные состояния в базисе Ваннье, полученные для 125000 волновых векторов в полной зоне Бриллюэна. Поскольку имеющиеся экспериментальные данные указывают на электронный характер проводимости, в учет были взяты только зонные состояния в интервале $\pm 0.5 \mathrm{eV}$ от уровня Ферми, соответствующего полученным в экспериментах значениям концентрации носителей. Соответствующие плотности состояний вблизи дна зоны проводимости представлены на рис. 3.

Особенностью расчетов по методу [6] является то, что вероятности электронных переходов между состояниями с волновыми векторами $\mathbf{k}$ и $\mathbf{k}+\mathbf{q}$, генерируемых за счет участия фононов с волновыми векторами q, вычисляются для большого набора случайно выбираемых векторов q. В наших расчетах набор q, в соответствии с рекомендациями авторов программы [6], включал 1000000 векторов.

\section{4. Результаты и обсуждение}

В данном разделе мы проводим сравнение рассчитанных транспортных характеристик с экспериментальными данными работ [22] для $\mathrm{CdO}$ и [25] для $\mathrm{SrTiO}_{3}$, в которых наиболее полно представлены зависимости транспортных свойств от концентрации носителей. В [22] при температурах отжига 700, 800, 900 и $1000 \mathrm{~K}$ концентрации носителей составляли соответственно $(0.75,2.51,3.75,4.28) \cdot 10^{19} 1 / \mathrm{cm}^{3}$. Концентрация примесей ниобия в $\mathrm{SrTiO}_{3}$ была от 2 до $6 \%$, чему соответствовала концентрация носителей $(4.79,5.98,7.98,9.57,10.30) \cdot 10^{19} 1 / \mathrm{cm}^{3}$. Расчеты транспортных характеристик выполнялись для данных концентраций.

На рис. 4 для $\mathrm{CdO}$, вместе с соответствующими экспериментальными данными, приведены температурные и концентрационные зависимости коэффицента Зеебека $S$, вычисленные по методикам [3] (код BoltzTraP-1) и [6] (Perturbo). (В силу кубической симметрии объектов $S=S_{x x}=S_{y y}=S_{z z}$, а недиагональные элементы тензора Зеебека пренебрежимо малы). Видно, что результаты, полученные по методике BoltzTraP-1, правильно отражают лишь температурную зависимость $S$. В отличие
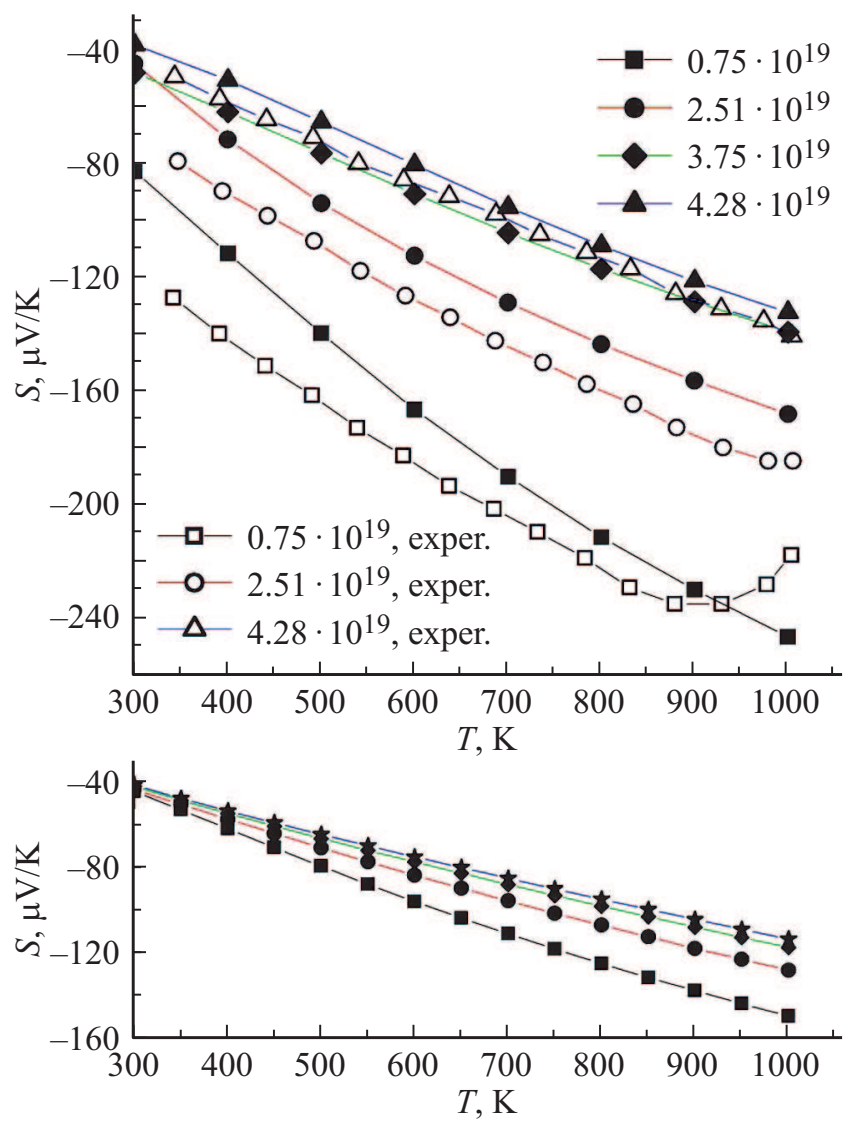

Рис. 4. Концентрационные и температурные зависимости коэффициента Зеебека оксида кадмия. Расчетные данные на верхней панели получены по методике [6], а на нижней - по методике [3]. Экспериментальные данные взяты из работы [22]. 

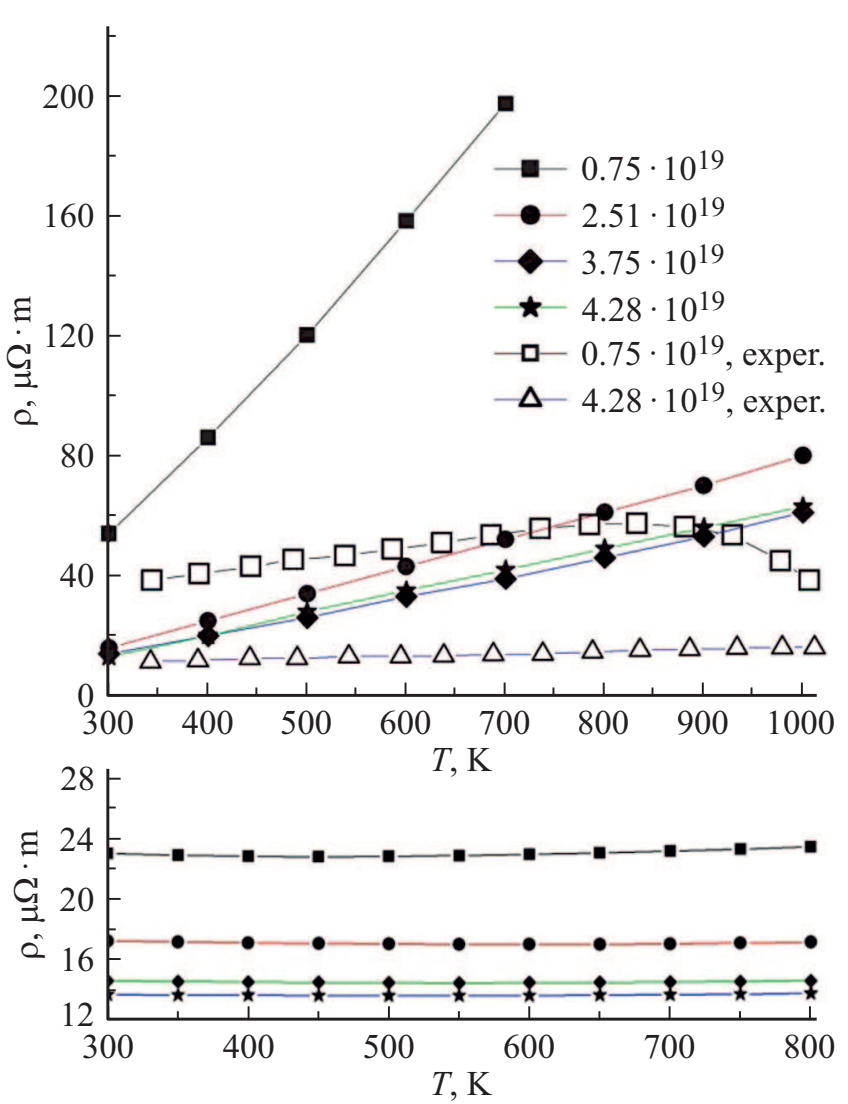

Рис. 5. Концентрационные и температурные зависимости удельного сопротивления оксида кадмия. Расчетные данные на верхней панели получены по методике Perturbo [6], а на нижней - по методике Boltztrap-1 [3]. Экспериментальные данные - работа [22].

от результатов эксперимента, их значения при $300 \mathrm{~K}$ почти не зависят от концентрации носителей и по абсолютному значению в среднем занижены. Результаты, полученные по методике Perturbo, как по абсолютному значению коэффициента Зеебека, так и по температурной зависимости, вплоть до $900 \mathrm{~K}$, хорошо соответствуют экспериментальным данным, в особенности для высоких концентраций носителей. Они также соответствуют наблюдаемому в эксперименте понижению абсолютной величины $S$ при возрастании концентрации носителей.

На рис. 5 приведены концентрационные и температурные зависимости удельного сопротивления $\mathrm{CdO}$ по двум методикам. Расчеты сопротивления по Boltztrap-1 [3] требуют независимой оценки времени релаксации. Мы выполнили эту оценку путем подгонки вычисленных для $T=300 \mathrm{~K}$ и вышеуказанных концентраций носителей значений $(\sigma / \tau)$ calc к имеющимся экспериментальным значениям сопротивления $\rho_{\text {exp }}$ при заданных концентрациях, что соответствует формуле $\tau=\frac{1}{\rho_{\exp }(\sigma / \tau)_{\text {calc }}}$. Усреднение по значениям концентрации носителей приводит к значению $\tau$ для оксида кадмия равному $7.6 \mathrm{fs}$. По сравнению с экспериментом, при температуре около $300 \mathrm{~K}$ расчетные значения сопротивления по обеим ме- тодикам, занижены, тогда как возрастание сопротивления с температурой по методике Perturbo преувеличено, a по Boltztrap-1 почти отсутствует. Отсутствие возрастания сопротивления по BoltzTraP-1 не может быть как-то оправдано. Однако при методике Perturbo заниженное значение сопротивления при $300 \mathrm{~K}$ и его чрезмерно высокий температурный коэффициент объясняется тем, что существенный вклад в сопротивление, видимо дает рассеяние на дефектах, в данном случае на кислородных вакансиях с зарядом +2 . Известно, что этот тип рассеяния пропорционален концентрации дефектов, но не зависит от температуры [28]. По правилу Маттсиессена [26]:

$$
\frac{1}{\tau}=\frac{1}{\tau_{e-p h}}+\frac{1}{\tau_{e-d e f}}
$$

при сложении скорости электрон-фононной релаксации $(1 / \tau)$, определяемой временем $\tau_{e-p h}$ со скоростью электрон-дефектной релаксации, зависящей от $\tau_{e-d e f}$, температурный коэффициент полного времени релаксации $\tau$, т. е. и сопротивления, должен уменьшаться. Видно также, что температурный коэффициент сопротивления понижается с увеличением концентрации вакансий, что согласуется с усилением рассеяния на вакансиях. Ниже мы покажем, что заключение о важной роли взаимодействия с дефектами соответствует и результатам по $\mathrm{SrTiO}_{3}$.

На рис. 6 приведены расчетные и экспериментальные данные [25] для коэффициента Зеебека $\mathrm{SrTiO}_{3}$.

Видно, что результаты, полученные по методике Boltzmann-1, хорошо воспроизводят температурную зависимость коэффициента Зеебека, но завышены более

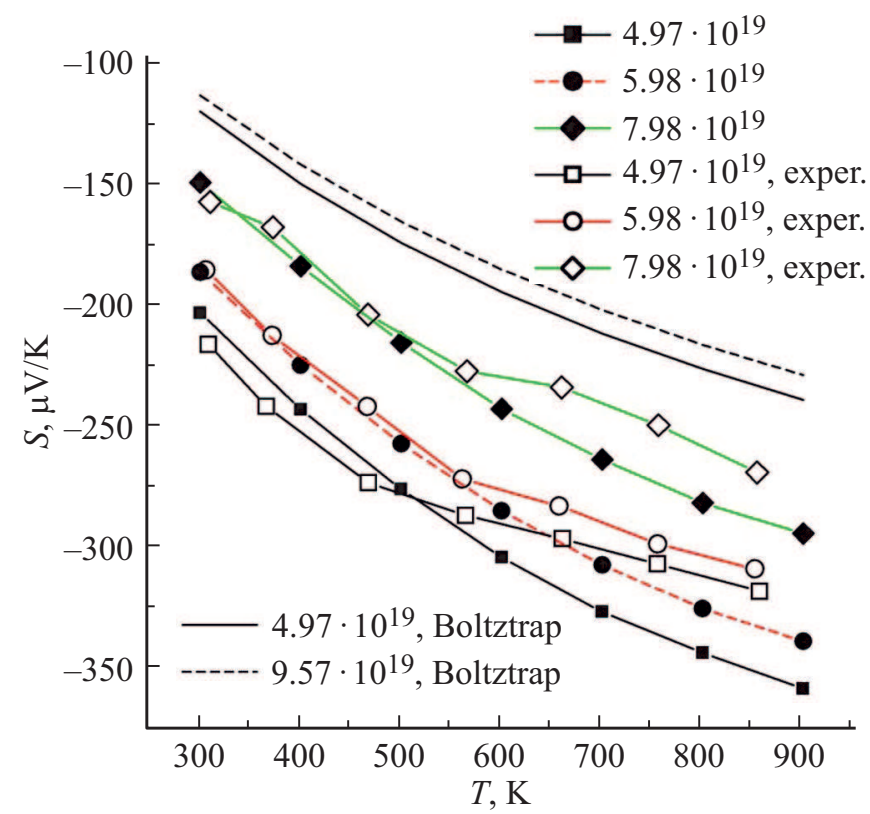

Рис. 6. Концентрационные и температурные зависимости коэффициента Зеебека $\mathrm{SrTiO}_{3}$. Расчетные данные получены по методике Perturbo [6] и по методике Boltzman-1 [3]. Экспериментальные данные - из работы [25]. 


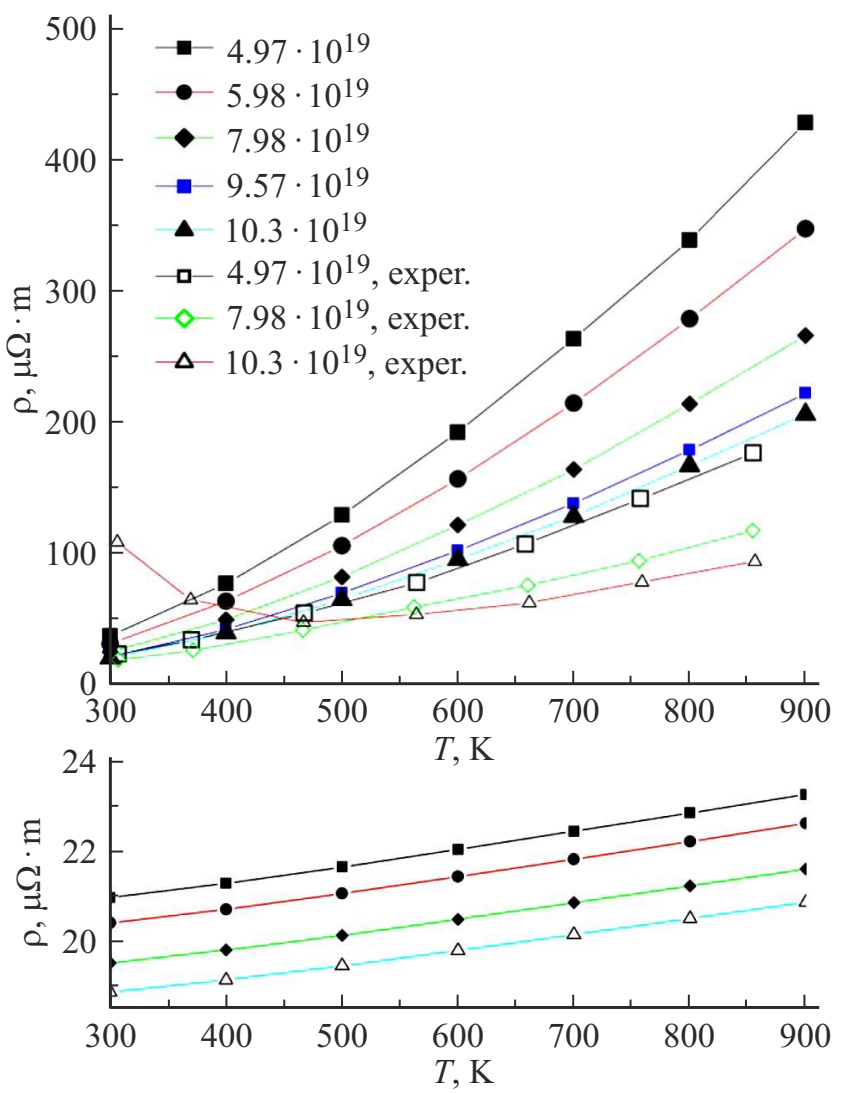

Рис. 7. Концентрационные и температурные зависимости удельного сопротивления $\mathrm{SrTiO}_{3}$. Расчетные данные на верхней панели получены по методике Perturbo [6], а на нижней по методике Boltzman-1 [3]. Экспериментальные данные см. раб. [25].

чем на $50 \mu \mathrm{V} / \mathrm{K}$ и не отражают зависимости $S$ от концентрации носителей. Наоборот, результаты, полученные по методике Perturbo, вплоть до $\sim 500 \mathrm{~K}$ почти идеально соответствуют экспериментальным зависимостям от концентрации и температуры. При $T>500 \mathrm{~K}$ экспериментальные данные отклоняются от закономерностей, представленных экспериментальными данными при более низких температурах, что, по-видимому, свидетельствует о наличии при высоких температурах изменений параметров кристаллической структуры. Данное предположение находит оправдание в результатах работы [47], где было показано, что в образцах $\mathrm{SrTiO}_{3}$, полученных методами электронно-лучевого испарения и микроволнового распыления, при повышении температуры от 500 до $600 \mathrm{~K}$ имеет место трехкратное понижение размеров кристаллитов и понижение периода решетки на $0.3 \%$.

На рис. 7 показаны теоретические и экспериментальные значения удельного сопротивления $\mathrm{SrTiO}_{3}$. При расчетах по методике Boltztrap-1 время релаксации было оценено по такой же процедуре, что и для оксида кадмия; оно оказалось равным 3.2 fs. Результаты, полученные по данной методике, демонстрируют, в противоречие эксперименту, очень слабую зависимость удельного сопротивления - как от концентрации носителей, так и температуры. Что касается методики Perturbo, то при $300 \mathrm{~K}$ полученные результаты при всех концентрациях носителей, за исключением максимальной, находятся в удовлетворительном соответствии с экспериментальными данными. Так же, как и для $\mathrm{CdO}$, температурная зависимость сопротивления завышена, однако, степень ее завышения значительно меньше. По аналогии со случаем оксида кадмия, можно предположить, что данное рассогласование объясняется отсутствием учета в наших расчетах рассеяния носителей на примесях. Согласно [28], скорость рассеяния на дефектах пропорциональна квадрату их эффективного заряда. Отсюда следует, что рассеяние на примесях ниобия ориентировочно в 4 раза слабее рассеяния на кислородных вакансиях. Это обстоятельство вполне согласуется с меньшим отклонением вычисленных для $\mathrm{SrTiO}_{3}$ температурных зависимостей сопротивления от эксперимента, чем в случае CdO. При увеличении концентрации примеси ниобия рассчитанная температурная зависимость понижается и приближается к экспериментальной, что, как и в случае $\mathrm{CdO}$, также может быть объяснено усилением рассеяния на примесях, не зависящем от температуры.
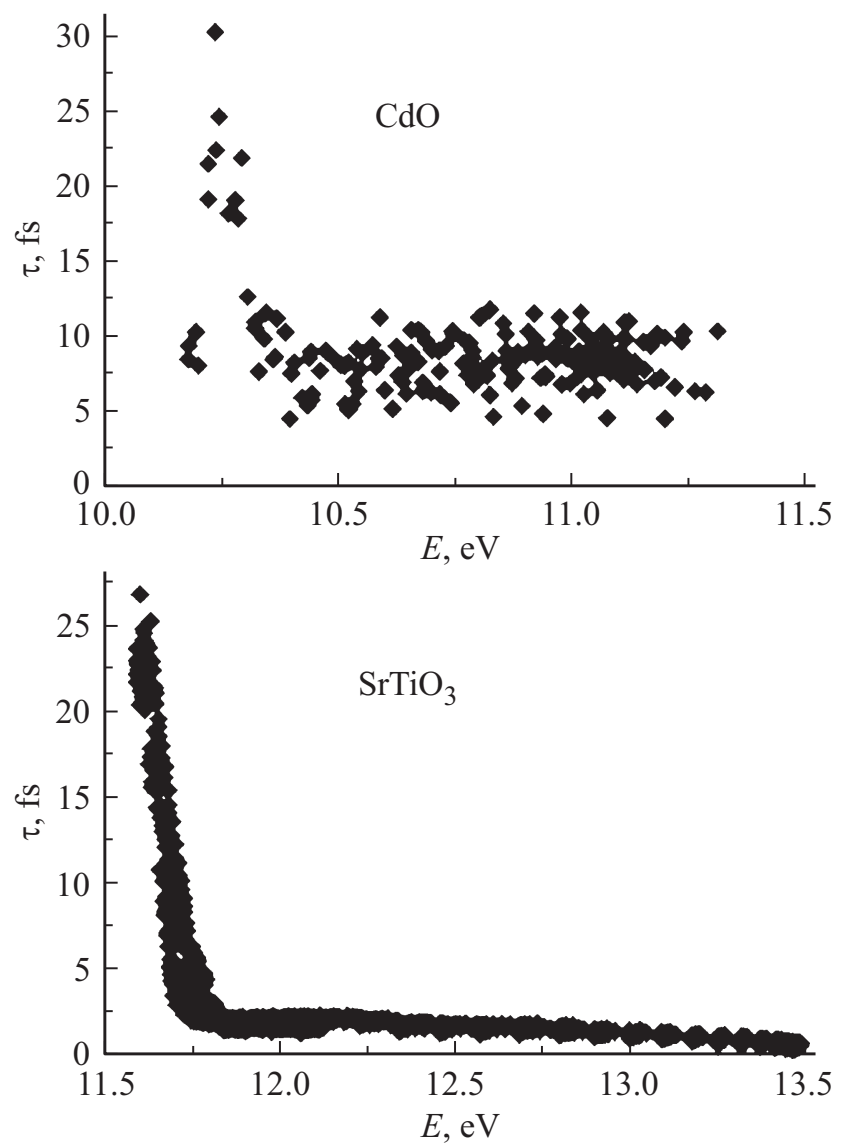

Рис. 8. Вычисленные значения времени электрон-фононной релаксации для $\mathrm{CdO}$ и $\mathrm{SrTiO}_{3}$ в зависимости от энергии электронных состояний. 

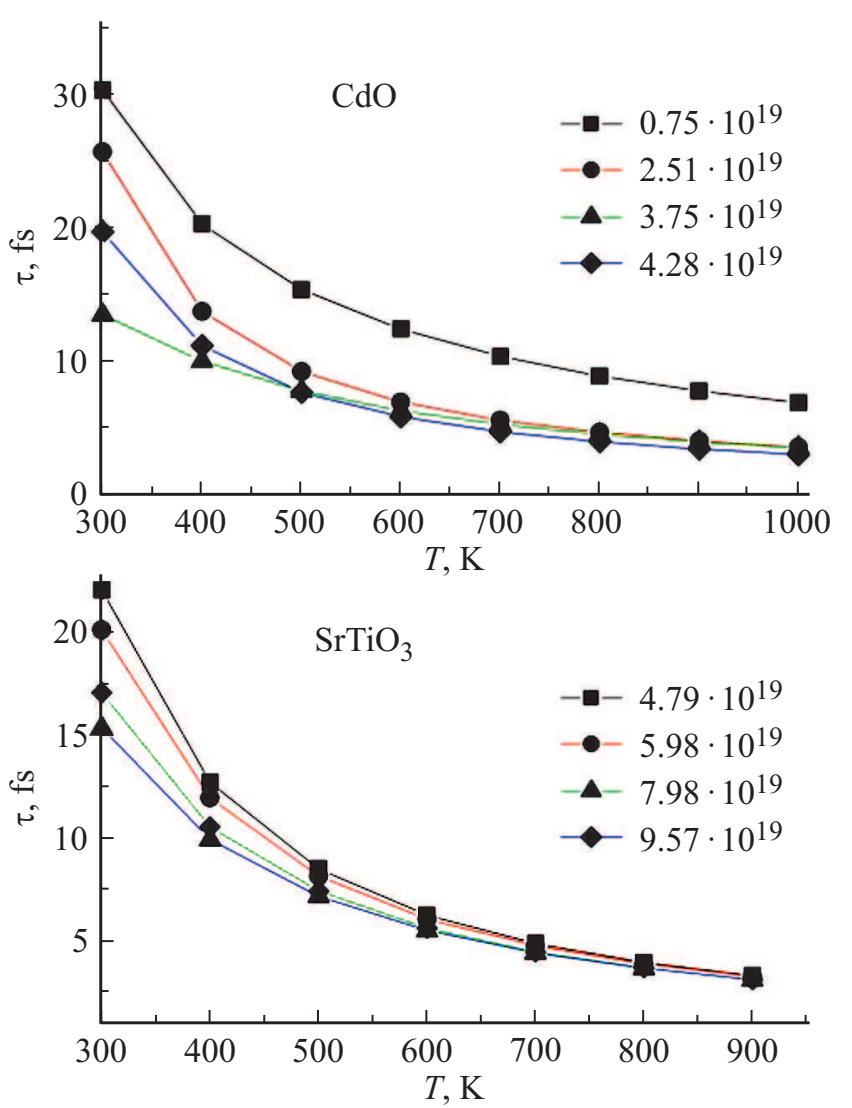

Рис. 9. Температурные зависимости времени релаксации для электронных состояний с энергиями, максимально близкими к уровню Ферми, вычисленные для $\mathrm{CdO}$ и $\mathrm{SrTiO}_{3}$.

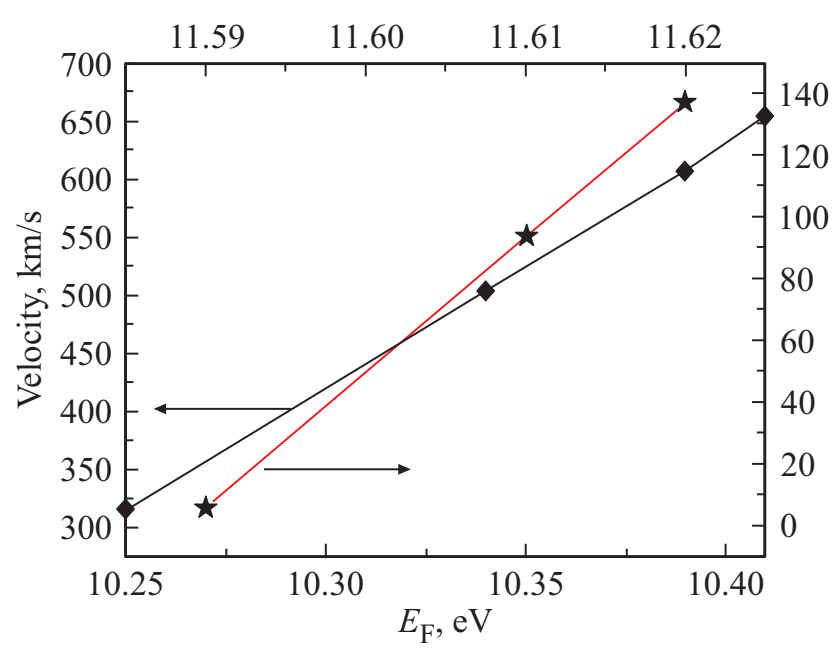

Рис. 10. Зависимости средней скорости электронов на уровне Ферми от энергии Ферми.

В целом, расчеты сопротивления по методике Perturbo [6] приводят к более качественным результатам, чем по методике Boltzman-1 [3], в особенности, для удельного сопротивления. Наиболее существенной погрешностью в результатах расчетов по методике Boltzman-1 является почти полное отсутствие зависи- мости сопротивления от температуры и концентрации дефектов. Поскольку главное отличие методики [3] от методики [6] заключается в использовании в первом случае приближения фиксированного времени релаксации, это ставит под сомнение эффективность использования этого приближения. На рис. 8 мы приводим данные по времени электрон-фононной релаксации в $\mathrm{CdO}$ и $\mathrm{SrTiO}_{3}$, вычисленные для электронных состояний вблизи дна зоны проводимости при температуре $300 \mathrm{~K}$.

В зависимости от концентрации носителей и температуры уровень Ферми смещается в диапазоне $10.15-10.25 \mathrm{eV}$ для оксида кадмия и $11.41-11.61$ для $\mathrm{SrTiO}_{3}$, т.е. в обоих случаях он находится в диапазоне резкого изменения времени релаксации. Лишь при энергии более $\sim 10.5 \mathrm{eV}$ для $\mathrm{CdO}$ и более $\sim 11.7$ для $\mathrm{SrTiO}_{3}$ среднее время релаксации можно считать постоянным, однако, это может иметь место при концентрации носителей значительно более высокой, чем достижимая в экспериментах. Отсутствие учета зависимости времени релаксации от энергии Ферми в методике BoltzTrap-1 является причиной отсутствия зависимости сопротивления от концентрации носителей.

Поскольку скорость электронов в уравнениях (6)-(9) не зависит от температуры, наблюдаемое в большинстве случаев в эксперименте возрастание сопротивления с ростом температуры, вероятно, связано с понижением времени релаксации. На рис. 9 показаны зависимости от температуры и концентрации носителей для времени релаксации электронных состояний $\mathrm{CdO}$ и $\mathrm{SrTiO}_{3}$, энергия которых наиболее близка к уровню Ферми, вычисленные по методике Perturbo. Для всех концентраций имеется понижение времени релаксации с температурой, коррелирующее с температурным возрастанием сопротивления. Однако, при любой фиксированной температуре с повышением концентрации носителей время релаксации понижается, что противоречит понижению сопротивления при этой температуре. Одним из факторов, увеличивающим проводимость при повышении концентрации носителей, т. е. уменьшающим сопротивление, является повышение плотности состояний на уровне Ферми, очевидное на рис. 3.

Другим фактором, ответственным за уменьшение сопротивления, является возрастание скорости электронов на уровне Ферми. Согласно рис. 10, оно имеет место и для $\mathrm{CdO}$, и для $\mathrm{SrTiO}_{3}$ для оксида кадмия (ромбы) и титаната стронция (звездочки).

\section{5. Заключение}

На примере оксида кадмия, содержащего кислородные вакансии, и титаната стронция, допированного ниобием, мы оценили степень корректности расчетов коэффициента Зеебека и удельного сопротивления по двум методиками „из первых принципов“, основанных на основе теории Больцмана и реализованных в программных комплексах Boltztrap-1 [3] и Perturbo [6]. Из расчетов 
следует, что методика Boltztrap-1 имеет ограниченную точность: полученные значения коэффициента Зеебека занижены по абсолютной величине и не воспроизводят наблюдаемые в эксперименте зависимости от температуры и концентрации носителей. К более правильным результатам приводят расчеты по методике Perturbo, которая включает оценку времени релаксации „из первых принципов“. Выполненные расчеты времени электронфононной релаксации указывают на то, что как для $\mathrm{CdO}$ так и $\mathrm{SrTiO}_{3}$ характерна существенная зависимость времени релаксации от концентрации носителей и температуры.

Пренебрежение этими зависимостями в методике Boltztrap-1, по-видимому, является главной причиной нивелирования зависимости коэффициента Зеебека и удельного сопротивления от концентрации носителей и температуры. Для $\mathrm{CdO}$ методика Perturbo почти во всех случаях приводит к хорошему согласию с экспериментом. В случае $\mathrm{SrTiO}_{3}$ эта же методика при температуре до $500 \mathrm{~K}$ приводит к почти идеальному согласию вычисленного коэффициента Зеебека с экспериментальными данными. Успех расчета коэффициента Зеебека связан, по-видимому, с тем, что, согласно уравнению (11), при его вычислении происходит компенсация погрешностей в расчете времени релаксации вследствие деления тензоров $L^{(1)} / L^{(0)}$. Наблюдаемые отклонения расчетных данных от экспериментальных при температуре выше $500 \mathrm{~K}$ связаны, вероятно, с фиксируемыми в экспериментах изменениями в периоде решетки.

Однако расчеты по методике Perturbo приводят с завышенной зависимости удельного сопротивления от температуры. Сопоставление результатов для $\mathrm{CdO}$ и $\mathrm{SrTiO}_{3}$ позволяет заключить, что этот недостаток связан с неучетом рассеяния носителей на дефектах, т.е. кислородных вакансиях в $\mathrm{CdO}$ и примесях $\mathrm{Nb}$ в $\mathrm{SrTiO}_{3}$. Заметим, что разработка компьютерных кодов для расчета „из первых принципов“ времени релаксации носителей на ионизированных дефектах является актуальной задачей, полностью еще не решенной. Так, авторами методики Perturbo была опубликована работа по рассеянию на вакансиях в кремнии [48], однако развитие этой работы не последовало. Другая методика, включающая рассеяние на ионизированных примесях, предложена авторами [49], однако тестирование методики на допированных полупроводниках не проводилось.

Обращает на себя внимание невоспроизводимое в расчетах аномальное изменение измеренных коэффициента Зеебека и сопротивления оксида кадмия с концентрацией носителей $0.75 \cdot 10^{19} 1 / \mathrm{cm}^{3}$ при температуре $800-1000 \mathrm{~K}$, свидетельствующее о переходе при этой температуре к полупроводниковому характеру проводимости. Для допированного $\mathrm{SrTiO}_{3}$ полупроводниковая проводимость также наблюдается при максимальной концентрации носителей, $4.79 \cdot 10^{19} 1 / \mathrm{cm}^{3}$ и температуре от 300 до $500 \mathrm{~K}$. Вероятно, отклонение результатов расчета от данных аномалий связано с тем, что в расчетах учитывались лишь электроны зоны проводимости, возникающие при появлении вакансий или допировании. Из-за высокой плотности состояний в валентной зоне учет их вкладов в проводимость требует несравнимо более высоких компьютерных ресурсов, чем при учете вкладов только электронов зоны проводимости.

\section{Финансирование работы}

Работа проведена за счет средств госбюджета. Все вычисления были выполнены на кластере URAN Института математики и механики УрО РАН.

\section{Конфликт интересов}

Авторы заявляют, что у них нет конфликта интересов

\section{Список литературы}

[1] F. Giustino, M. L. Cohen, S.G. Louie. Phys. Rev. B 76, 165108 (2007).

[2] F. Giustino. Rev. Mod. Phys. 89, 015003 (2017).

[3] G.K.H. Madsen, D.J. Singh. Comp. Phys. Commun. 175, 67 (2006).

[4] G.K.H. Madsen, J. Carrete, M.J. Verstraete. Comp. Phys. Commun. 231, 140 (2018).

[5] T.J. Scheidemantel, C. Ambrosch-Draxl, T. Thonhauser, J.V. Badding, J.O. Sofo. Phys. Rev. B 68, 125210 (2003).

[6] J.-J. Zhou, J. Park, I-T. Lu, I. Maliyov, X. Tong, M. Bernardi. Comp. Phys. Commun. 264, 107970 (2021).

[7] G. Kresse, M. Marsman, J. Furthmüller. Vienna ab-initio simulation package. VASP the guide. UniversitätWien, Wien (2018). 233 p.

[8] P. Giannozzi, O. Andreussi, T. Brumme. J. Phys.: Condens. Matter. 29, 465901 (2017).

[9] P. Blaha, K. Schwarz, F. Tran, R. Laskowski, G.K.H. Madsen, L.D. Marks. J. Chem. Phys. 152, 074101 (2020).

[10] S. Ponce, E.R. Margine, C. Verdi, F. Giustino. arXiv:1604.03535

[11] J. Noffsinger, F. Giustino, B.D. Malone, Ch-H. Park, S.G. Louie, M.L. Cohen. Comp. Phys. Commun. 181, 2140 (2010).

[12] A. Faghaninia. Theory of Carrier Transport From First Principles: Applications in Photovoltaic and Thermoelectric Materials. Dissertation. University of Washington (2016). $219 \mathrm{p}$.

[13] Y. Wang, Sh.-L. Shang, H. Fang, Z.-K. Liu, L.-Q. Chen. Comp. Mater. 2, 16006 (2016).

[14] F. Ricci, W. Chen, U. Aydemir, G.J. Snyder, G.-M. Rignanese, A. Jain, G. Hautier. Scientific Data 4, 17008 (2018).

[15] M. Yasukawa, K. Ueda, S. Fujitsu, H. Hosono. Ceram. Int. 43, 9653 (2017).

[16] L. Lindsay, D.S. Parker. Phys. Rev. B 92, 144301 (2015).

[17] A.A. Adewale, A. Chik, R.M. Zaki, F.Ch. Pa, Y.Ch. Keat, N.H. Jamil. Int. J. Nanoelectron. Mater. 12, 477 (2019).

[18] M.U. Kahaly, U. Schwingenschlogl. J. Mater. Chem. A 2, 10379 (2014).

[19] A. Mukasia, G.S. Manyali, H. Barasa, J. Sifuna. J. Mater. Sci. Res. Rev. 2, 1 (2019). 
[20] S.K. Vasheghani Farahani, V. Munoz-Sanjose, J. Zuniga-Perez, C.F. McConville, T.D. Veal. Appl. Phys. Lett. 102, 022102 (2013).

[21] L. Qing, W.Sh. Fang, L.L. Jiang, W.J. Long, D.Sh. Yu, Y. Wei, F.G. Sheng. Sci. China-Phys. Mech. Astron 57, 1644 (2014).

[22] X. Zhang, H. Li, J. Wang. J. Adv. Ceram. 4, 226 (2015).

[23] Y. Cui, J.R. Salvador, J. Yang, H. Wang, G. Ampw, H. Klainke. J. Electron. Mater. 38, 1002 (2009).

[24] A.V. Kovalevsky, A.A. Yaremchenko, S. Populoh, A. Weidenkaff, J.R. Frade. J. Appl. Phys. 113, 053704 (2013).

[25] T.T. Khan, S.-Ch. Ur. Electron. Mater. Lett. 14, 336 (2018).

[26] N.W. Ashcroft, N.D. Mermin. Solid State Physic. Cengage Learning (2011). 833 p.

[27] Ph.B. Allen. Boltzmann. Boltzmann theory and resistivity in metals. In: Quantum Theory of Real Materials. Kluwer, Boston (1996). P. 219.

[29] C. Jacoboni. Theory of electron transport in semiconductors. Springer series in solid-state sciences. Berlin-Heidelberg, Springer (2010). $588 \mathrm{p}$.

[29] K. Burke. J. Chem. Phys. 136, 150901 (2012).

[30] K. Zeeger. Semiconductor Physics: An introduction. Springer (1991). $538 \mathrm{p}$.

[31] A. Migdal. Sov. Phys. JETP 34, 996 (1958).

[32] G.M. Eliashberg. Sov. Phys. JETP 11, 696 (1960).

[33] S. Baroni, S. de Gironcoli, A. Dal Corso. Rev. Mod. Phys. 73, 515 (2001).

[34] G. Kresse, D. Joubert. Phys. Rev. B 59, 1758 (1999).

[35] J. Paier, R. Hirschl, M. Marsman, G. Kresse. J. Chem. Phys. 122, 234102 (2005).

[36] J.P. Perdew, A. Ruzsinszky, G.I. Csonka, O.A. Vydrov, G.E. Scuseria, L.A. Constantin, X. Zhou, K. Burke. Phys. Rev. Lett. 100, 136406 (2008).

[37] A.M. Rappe, K.M. Rabe, E. Kaxiras, J.D. Joannopoulos. Phys. Rev. B 41, 1227 (1990).

[38] D. Vanderbilt. Phys. Rev. B 41, 7892 (1990).

[39] J.P. Perdew, A. Zunger. Phys. Rev. B 23, 5048 (1981).

[40] B. Himmetoglu, A. Janotti, H. Peelaers, A. Alkauskas, Ch.G. Van de Walle. Phys. Rev. B 90, 241204 (2014).

[41] R. Cuscó, J. Ibáñez, N. Domenech-Amador, L. Artús, J. Zúñiga-Pérez, V. Muñoz-Sanjosé. J. Appl. Phys. 107, 063519 (2010).

[42] M. Cococcioni, S. de Gironcoli. Phys. Rev. B 71, 035105 (2005).

[43] O. Madelung. Landolt-Bornstein Numerical Data and Functional Relationships. Springer, Berlin (1984). V. 17. 666 p.

[44] L. Lindsay, D.S. Parker. Phys. Rev. B 92, 144301 (2015).

[45] K. van Benthem, C. Elsässer. J. Appl. Phys. 90, 6156 (2001).

[46] I. Souza, N. Marzari, D. Vanderbilt. Phys. Rev. B 65, 035109 (1997).

[47] F. Hanzig, J. Hanzig, E. Mehner, C. Richter, J. Vesely, H. Stocker, B. Abendroth, M. Motylenko, V. Klemm, D. Novikov, D.C. Meyera. J. Appl. Crystallography 48, 393 (2015).

[48] I.-T. Lu, J. Park, J.-J. Zhou, M. Bernardi. Comp. Mater. 6, 17 (2020).

[49] A.M. Ganose, J. Park, A. Faghaninia, R. Woods-Robinson, K.A. Persson, A. Jain. Nature Commun. 12, 1 (2021).

Редактор Т.Н. Василевская 